\title{
Isoquercetin ameliorates myocardial infarction through anti-inflammation and anti-apoptosis factor and regulating TLR4-NF-кB signal pathway
}

\author{
CHENGTAI MA ${ }^{1}$, YANXIA JIANG ${ }^{2}$, XIAOHUI ZHANG ${ }^{1}$, XIAOXUE CHEN $^{1}$, ZHENFANG LIU $^{1}$ and XINTAO TIAN ${ }^{1}$ \\ Departments of ${ }^{1}$ Emergency and ${ }^{2}$ Pathology, The Affiliated Hospital of Qingdao University, \\ Qingdao, Shandong 266003, P.R. China
}

Received February 20, 2017; Accepted September 27, 2017

DOI: $10.3892 / \mathrm{mmr} .2018 .8709$

\begin{abstract}
The aim of the present study was to investigate the protective mechanisms and identify the effects of isoquercetin on myocardial infarction in a rat model of acute myocardial infarction (AMI). Isoquercetin ameliorated myocardial infarct size, creatine kinase (CK), CK-MB and lactic dehydrogenase activity and inhibited inflammation, oxidative stress and heart cell apoptosis in a rat with AMI. Isoquercetin increased endothelial nitric oxide synthase, reduced inducible nitric oxide synthase levels and suppressed the Toll-like receptor 4-nuclear factor (TLR4-NF)- $\kappa \mathrm{B}$ signaling pathway in a rat with AMI. Overall, isoquercetin ameliorated AMI through anti-inflammatory and anti-apoptotic factors, and regulation of the TLR4-NF- $\kappa \mathrm{B}$ signaling pathway. Isoquercetin may therefore potentially exert a protective effect against AMI or other heart diseases.
\end{abstract}

\section{Introduction}

As the aging of population rises, the heart failure has become a more and more serious social issue (1). It is true that the heart failure is the final battlefield of nearly all heart diseases such as IHD, valvular heart disease, hypertension, myocarditis, cardiomyopathy and diabetes developing to their terminal stage $(1,2)$. But it is also widely acknowledged that various pathological changes after the myocardial infarction, like changes in the myocardial cell morphology, ECM remodeling, microvascular insufficiency and ventricular dilatation, take up an important place in the occurrence and development of the heart failure (3).

Correspondence to: Professor Yanxia Jiang, Department of Pathology, The Affiliated Hospital of Qingdao University, 16 Jiangsu Road, Qingdao, Shandong 266003, P.R. China

E-mail: chingyx93@126.com

Key words: isoquercetin, acute myocardial infarction, inflammation, oxidative stress, TLR4-NF- $\mathrm{B}$
Myocardial infarction: Due to the effects of multiple elements including mechanical stress, endocrine stimulation and cytokine activation, the ventricular form and structure have both changed, leading to a ventricular remodeling (4). Such a change could affect both the infarct area and the non-infarct area, demonstrating a thinner, extended or dilated ventricular wall in the infarct area (2). While the ventricular wall in the non-infarct area is thickened and extended reactively, eventually causing the ventricular dilatation and cardiac insufficiency (5).

The inflammatory responses of heart could be summarized as pure native immune reaction and/or the combination of the native immune reaction and acquired immune reaction (6). The most typical feature of the native immune reaction is to induce the production of inflammatory factors. When myocardial ischemia occurs and even causes the heart failure, usually the native immune reaction and inflammatory responses both occur (7).

Myocardial infarction could cause the elevation in the oxidative stress level of myocardium and further result in the oxidative damage on the myocardium (8). As a secondary messenger, ROS could deliver the stress signals and make various effects on cells. Proper ROS level could effectively activate cells' redox sensitive signal channels such as MAPKs and PI3K/AKt, with each channel being interconnected (9). MAPKs and PI3K/AKt adjust the transcription factors like HSF-1, P53 and NF- $\kappa$ B through phosphorylation, thus inducing the expression of a series of redox sensitive genes, to control the reactions of signals such as growth, apoptosis or stress (8). However, overdose of ROS could cause the cell apoptosis or necrosis. It is universally accepted that the myocardial infarction will cause the oxidative stress and lead to the pathologic myocardial remodeling and myocardial enlargement (10).

TLRs expressed by the myocardial cells are majorly TLR2, TLR3 and TLR4. According the combined protein, the TLRs signal channels could be separated into two types: MyD88 channel and Trif channel (11). MyD88 channel could activated by all TLRs except TLR3 while Trif channel could be activated by TLR3 and TLR4. These two channels could both activate NF- $\mathrm{NB}$ eventually, which is a pivot transcription factor for activating the inflammatory responses (12). It has been reported that TLRs have played a crucial rule in inducing 
the inflammatory responses of heart diseases like myocardial infarction and viral myocarditis (13).

Limonium sinense (Girard) Kuntze belongs to the genus of Limonium, the family of plumbaginaceae, also called sea spinach, sea Radix Paeoniae Rubra, spoon-like leaf grass or salt cloud grass (14). As a perennial salt-excretion medical herb, it is listed as the protected plant species by China in 2001 (15). Chinese Limonium sinense possesses the function of clearing heat and removing toxicity, removing blood stasis and hemostasis, dispelling the wind and relieving inflammation as well as anticancer and anti-aging. Studies have suggested that flavone is the one of the major effective ingredients in the Chinese Limonium sinense and abundant literatures have attested that isoquercetin (Fig. 1) and morin are both found to be engaged in the biological activities like anti-inflammation, antioxidant and decompression; quercetin is identified with the function of antioxidant, anticancer and antimutagen; luteolin possesses the functions of controlling the proliferation of cancer cells and inducing the cancer cells to apoptosis or the sensitization of the apoptosis; and apigenin has been already found to be clearly active in the antitumor in a mechanism $(16,17)$. These are all the major flavone active ingredients in the Traditional Chinese Medicines (TCM) and the important indexes to evaluate the quality of medicines (18). The aim of the present study was to reveal the protective mechanisms and identify the effects of isoquercetin on myocardial infarction in a rat model of acute myocardial infarction.

\section{Materials and methods}

Animals, experimental design and protocol. Male Sprague-Dawley (SD) rats (220-250 g, 6-8 weeks) were provided by Qingdao University and housed in separated cages with laboratory chow and tap water ad libitum. SD rats were randomly allocated into three groups: Sham-operated control group (control, $\mathrm{n}=6$ ), myocardial infarction model group (MI model, $n=6$ ) and isoquercetin group (Iso, $n=6$ ). Control and model groups received an equal quantity of vehicle normal saline. SD rats were anesthetized $30 \mathrm{mg} / \mathrm{kg}$ pentobarbital and left anterior descending coronary artery was exposed and ligated with forceps at the proximal left anterior descending coronary artery 2-3 $\mathrm{mm}$ from its origin between the pulmonary artery conus. The heart was returned and the thorax was closed. Rat of Iso group was gavaged with $80 \mathrm{mg} / \mathrm{kg} /$ day isoquercetin for 2 weeks. The present study was approved by the Medical Ethics Committee of the Affiliated Hospital of Qingdao University (Qingdao, China).

Measurement of myocardial infarct size and histological analysis. Rat was sacrificed during anesthetization, and heart was toke out and washed with PBS. Heart tissue was transversely cut across the left ventricle and cutted $3 \mathrm{~mm}$ thick. Tissue samples were incubated with $0.5 \%$ TTC $0.5 \%$ (Amresco LLC, Solon, OH, USA) in phosphate buffer (Sangon Biotech Co., Ltd., Shanghai, China) at $37^{\circ} \mathrm{C}$ for $30 \mathrm{~min}$, and fixed with $10 \%$ formalin. For histological analysis, sections were stained with hematoxylin and eosin. The tissue was observed using a microscope (BX53; Olympus Corporation, Tokyo, Japan).<smiles>O=c1c(O[C@@H]2O[C@H](CO)[C@@H](O)[C@H](O)[C@H]2O)c(-c2ccc(O)c(O)c2)oc2cc(O)cc(O)c12</smiles>

Figure 1. Structural formula of isoquercetin.

Measurement of cardiac marker enzyme activity. Peripheral blood of rat was collected from eye socket during anesthetization and serum was collected after centrifuged at $1,000 \mathrm{x} \mathrm{g}$ for $20 \mathrm{~min}$. Serum was used to analyze creatine kinase (CK), CK-MB and LDH levels using commercial diagnostic kits (Shanghai Kehua Medical Instruments Co., Ltd., Shanghai, China).

Enzyme-linked immunosorbent assay (ELISA). Peripheral blood of rat was collected from eye socket during anesthetization and serum was collected after centrifuged at 1,000 $\mathrm{x} g$ for $20 \mathrm{~min}$. Serum was used to analyze inflammation and oxidative stress using ELISA kit.

Western blot analysis. Myocardial tissue $(80 \mathrm{mg}$ ) was grinded in liquid nitrogen and incubated with RIPA sassy and protein content was quantitated using BCA sassy. Protein/lane $(30 \mu \mathrm{g})$ was subjected by $8-12 \%$ SDS-PAGE and transferred onto nitrocellulose membranes (EMD Millipore, Billerica, MA, USA). Membranes were blocked with $5 \%$ bovine serum albumin in TBST and incubated with Bax (1:2,000), Bcl-2 (1:2,000), eNOS

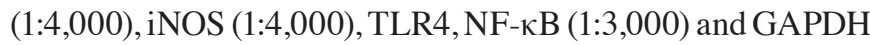
$(1: 5,000)$ (all from Cell Signaling Technology, Inc., Danvers, MA, USA) at $4^{\circ} \mathrm{C}$ overnight. Membranes were washed and incubated with anti-rabbit horseradish peroxidase-conjugated (1:5,000; Cell Signaling Technology, Inc.) in 5\% nonfat dry milk in washing buffer for $1 \mathrm{~h}$ at room temperature. Protein was detected using ECL sassy and calculated using Image-Pro Plus 6.0 software (Media Cybernetics, Inc., Rockville, MD, USA).

Statistical analysis. Data are reported as the mean \pm standard deviation. The data were statistically analyzed using a one-way ANOVA for multiple comparisons. $\mathrm{P}<0.05$ was considered to indicate a statistically significant difference.

\section{Results}

Isoquercetin ameliorates myocardial infarct size. At the end of the experiments, myocardial infarct size of MI rat was measured. We found that a significant increase of myocardial infarct size in MI model rat, compared with control group (Fig. 2A). However, treatment with isoquercetin significantly inhibited the increase of myocardial 

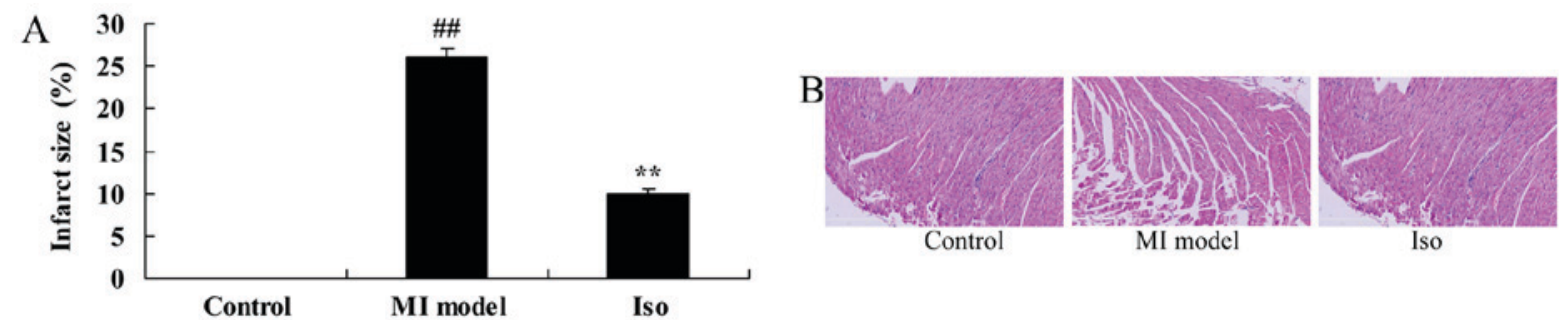

Figure 2. Isoquercetin ameliorates myocardial infarct size. Isoquercetin ameliorates myocardial infarct size (A) and H\&E staining (B). Control, control group; MI model, myocardial infarct model group; Iso, isoquercetin treatment group. ${ }^{\# \#} \mathrm{P}<0.01$ compared with control group, ${ }^{* * *} \mathrm{P}<0.01$ compared with myocardial infarct model group. H\&E, hematoxylin and eosin.
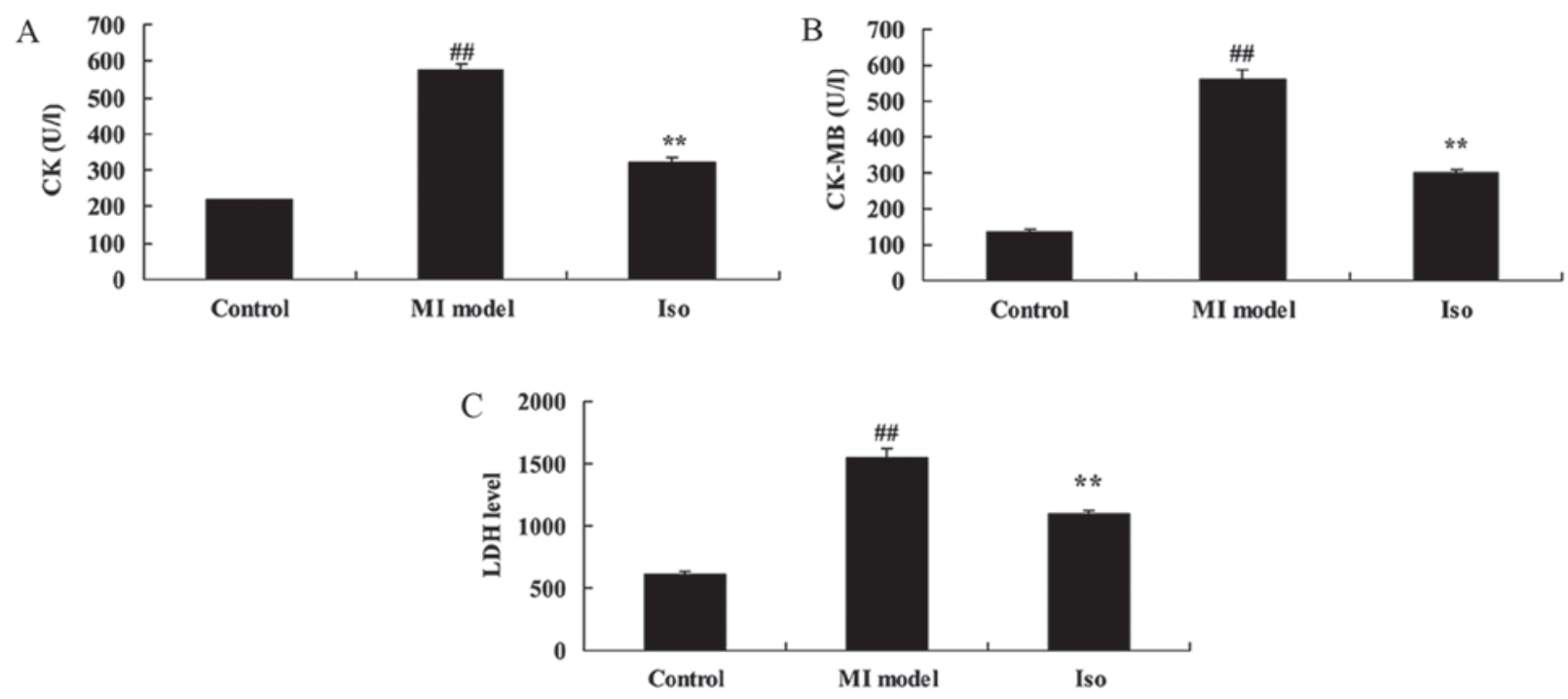

Figure 3. Isoquercetin ameliorates CK, CK-MB and LDH activity. Isoquercetin ameliorates CK (A), CK-MB (B) and LDH (C) activity. Control, control group; MI model, myocardial infarct model group; Iso, isoquercetin treatment group. ${ }^{\# *} \mathrm{P}<0.01$ compared with control group, ${ }^{* *} \mathrm{P}<0.01$ compared with myocardial infarct model group. CK, creatine kinase.

infarct size in MI rat (Fig. 2A). Meanwhile, hematoxylin and eosin (H\&E) staining showed that MI model rat appeared augmentation and loose arrangement, compared with control group (Fig. 2B). Treatment with isoquercetin significantly reduced augmentation and loose arrangement in MI rat (Fig. 2B).

Isoquercetin ameliorates $C K, C K-M B$ and $L D H$ activity. Instead, we report significant increases in CK, CK-MB and LDH activity of MI model rat, compared with control group (Fig. 3). Fig. 3 showed that isoquercetin significantly reduced $\mathrm{CK}, \mathrm{CK}-\mathrm{MB}$ and $\mathrm{LDH}$ activity in MI rat.

Isoquercetin inhibited inflammation. We explored the anti-inflammation effects of isoquercetin in MI model. The results of the ELISA assay indicate that IL- 6 and TNF- $\alpha$ levels increased significantly in the model group, compared with control group (Fig. 4). These elevations were significantly inhibited by isoquercetin in MI rat (Fig. 4).

Isoquercetin inhibited oxidative stress. We analyzed MDA and SOD levels in MI rat using ELISA kits. As showed in Fig. 5, the inhibition of SOD level and increase of MDA level were effectively observed in MI model group, compared with control group. After MI rat by isoquercetin, MDA level was inhibited and SOD level was increased in MI rat (Fig. 5).

Isoquercetin inhibited heart cell apoptosis. We probed that the anti-apoptosis effects of isoquercetin in MI rat, we measured caspase- 3 activity and $\mathrm{Bax} / \mathrm{Bcl}-2$ protein expression. Caspase-3 activity and $\mathrm{Bax} / \mathrm{Bcl}-2$ protein expression were significantly increased in MI model rat, compared with control group (Fig. 6). Isoquercetin significantly suppressed caspase- 3 activity and $\mathrm{Bax} / \mathrm{Bcl}-2$ protein expression in MI rat (Fig. 6).

Isoquercetin affects on NO levels. We sought iNOS and eNOS protein expression using Western blot analysis for cardioprotection. iNOS protein expression was significantly induced, and eNOS protein expression was significantly reduced in MI model rat, compared with control group (Fig. 7). Treatment with isoquercetin significantly suppressed iNOS protein expression and induced eNOS protein expression in MI rat (Fig. 7).

Isoquercetin affects on Toll-like receptor 4-nuclear factor (TLR4-NF)- $\kappa B$ signal pathway. The western blot analysis results indicated that TLR4 and NF- $\kappa B$ protein expression was 

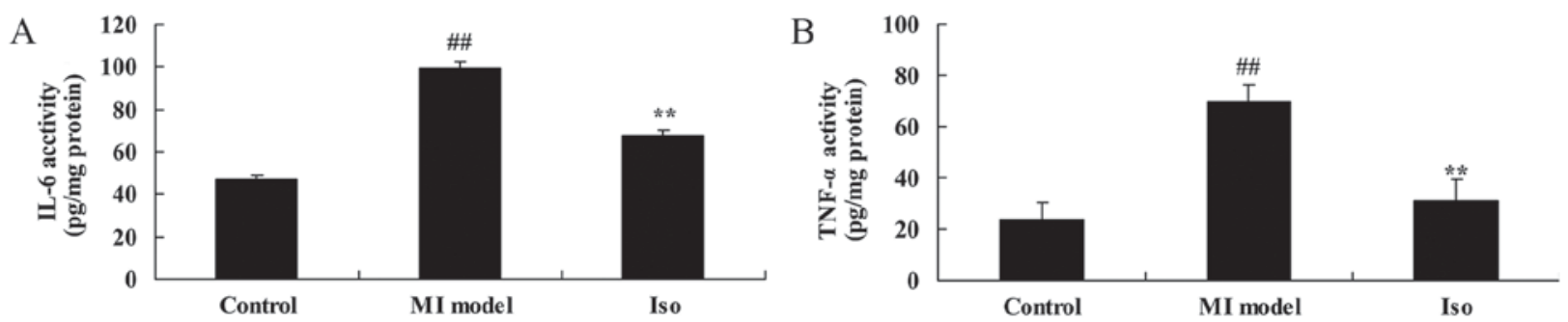

Figure 4. Isoquercetin inhibited inflammation. Isoquercetin inhibited IL-6 (A) and TNF- $\alpha$ (B) levels. Control, control group; MI model, myocardial infarct model group; Iso, isoquercetin treatment group. ${ }^{\# \#} \mathrm{P}<0.01$ compared with control group, ${ }^{* *} \mathrm{P}<0.01$ compared with myocardial infarct model group.
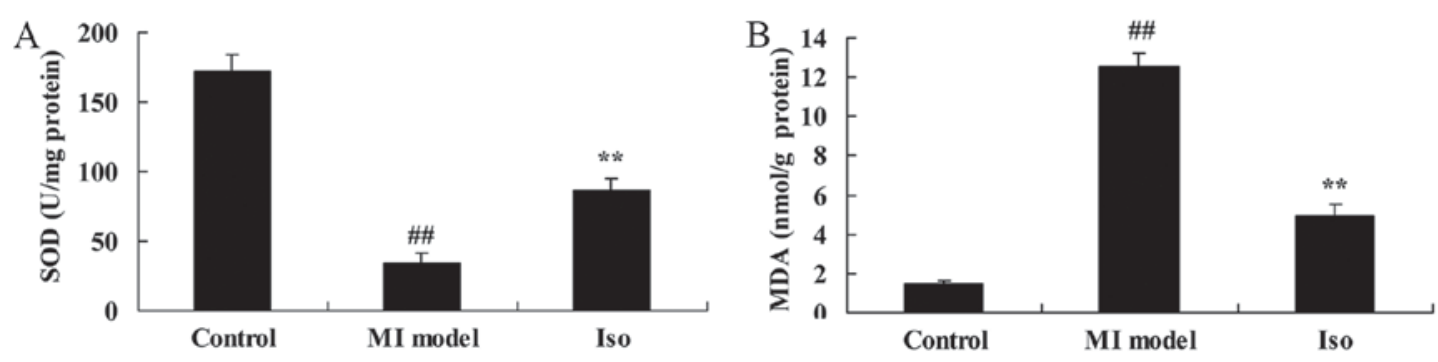

Figure 5. Isoquercetin inhibited oxidative stress. Isoquercetin increased SOD (A) and inhibited MDA (B) levels. Control, control group; MI model, myocardial infarct model group; Iso, Isoquercetin treatment group. ${ }^{\# \#} \mathrm{P}<0.01$ compared with control group, ${ }^{* *} \mathrm{P}<0.01$ compared with myocardial infarct model group.
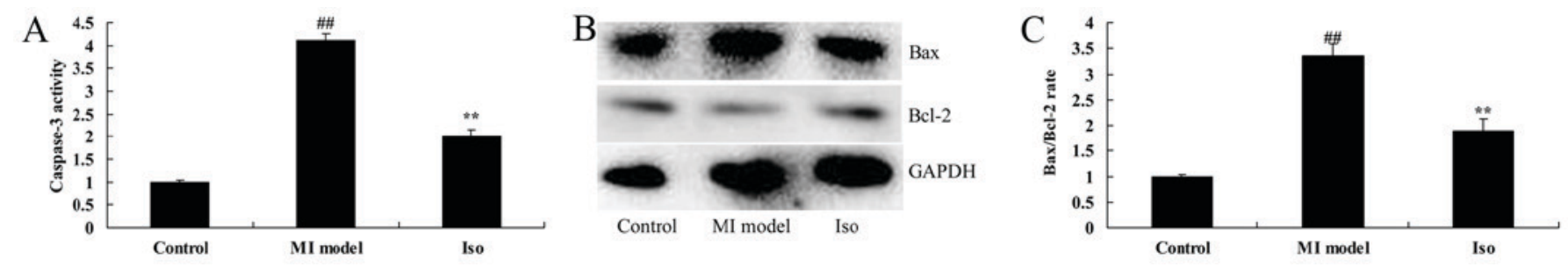

Figure 6. Isoquercetin inhibited heart cell apoptosis. Isoquercetin inhibited caspase-3 level (A) and Bax/Bcl-2 protein expression by western blotting assays (B) and statistical analysis (C). Control, control group; MI model, myocardial infarct model group; Iso, isoquercetin treatment group. ${ }^{\# \#} \mathrm{P}<0.01$ compared with control group, ${ }^{* *} \mathrm{P}<0.01$ compared with myocardial infarct model group.
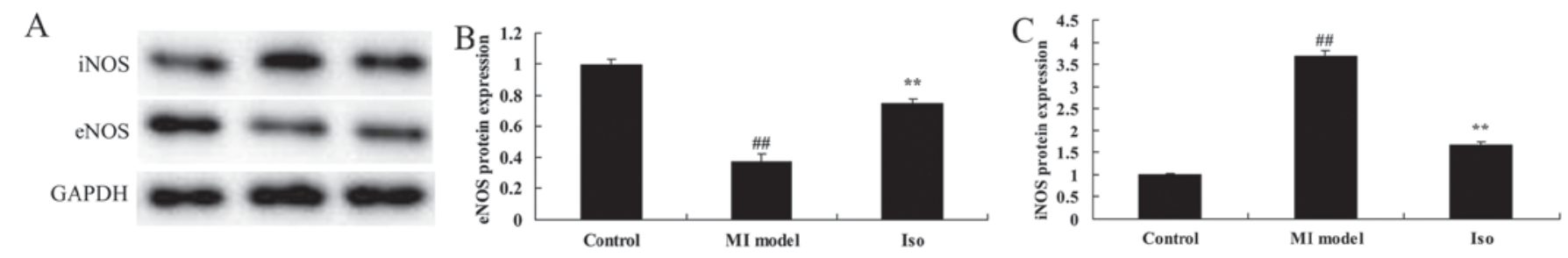

Figure 7. Isoquercetin affects on NO levels. Isoquercetin affects on iNOS and eNOS protein expression by western blotting assays (A) and statistical analysis (B and C). Control, control group; MI model, myocardial infarct model group; Iso, isoquercetin treatment group. ${ }^{\# \#} \mathrm{P}<0.01$ compared with control group, ${ }^{* *} \mathrm{P}<0.01$ compared with myocardial infarct model group.

significantly promoted in MI model group, compared with control group (Fig. 8). Isoquercetin significantly suppressed TLR4 and NF-KB protein expression in MI rat (Fig. 8).

\section{Discussion}

After myocardial infarction, the progressive enlargement and the shape change will occur to the ventricle, which is called the 'ventricular remodeling' after infarction, remaining as a major pathologic basis for the chronic heart failure after myocardial infarction (19). The ventricular remodeling includes the changes in the myocardial cells and the changes in the extracellular matrix (20). The dynamic balance between the synthesis and degradation of the fibrin collagen in the extracellular matrix plays an important role in the process of ventricular remodeling (21). The balance is broken after the myocardial infarction and leads to the myocardial interstitial fibrosis, limiting the myocardial activities and causing the decrease in the ventricular compliance and the myocardial contractility, which could further develop into the heart 
A

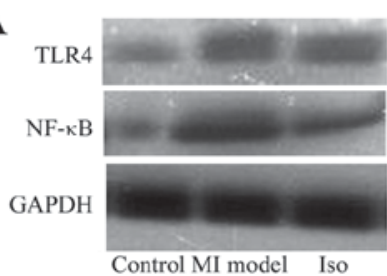

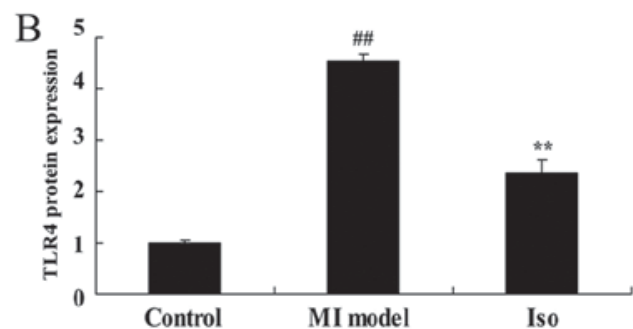

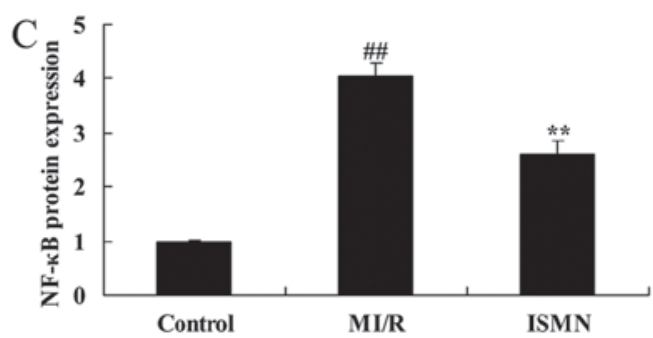

Figure 8. Isoquercetin affects on TLR4-NF- $\mathrm{B}$ signal pathway. Isoquercetin affects on LR4 and NF- $\kappa$ B protein expression by western blotting assays (A) and statistical analysis (B and C). Control, control group; MI model, myocardial infarct model group; Iso, isoquercetin treatment group. ${ }^{\# \# ~} \mathrm{P}<0.01$ compared with control group, ${ }^{* *} \mathrm{P}<0.01$ compared with myocardial infarct model group. TLR4-NF, Toll-like receptor 4-nuclear factor.

failure (22). Meanwhile, the collagen deposition could also slow down the evoked response passing through the myocardium, which damages the electrical stability of myocardial cells and leads to arrhythmia (3). Therefore, it was speculated for the purposes of the present study that isoquercetin significantly inhibited the increase of myocardial infarct size, and reduced CK, CK-MB and LDH activity in MI rat.

Myocardial infarction is the most common inducement for the chronic heart failure and even causes extensive inflammatory responses of the heart (23). Many studies, including this one, have observed that the expression of inflammatory factors in the ischemic area and distal area of the heart has increased after the myocardial infarction $(7,23)$. The inflammation in the infarct area could be explained as that the stress reaction occurring in the myocardial damage and concrescence in the infarct area causes the inflammation, accompanied by the infiltration of inflammatory cells (7). However, what is worth noticing is a report revealing that the distal area is still staying at an obvious inflammatory status for seven weeks since the myocardial infarction happened. In our study we observed isoquercetin significantly inhibited inflammation in a rat with acute myocardial infarction (AMI). Wang et al reported that isoquercetin ameliorates via anti-oxidative, anti-inflammatory, and anti-apoptotic effects in cerebral impairment (14).

Multiple studies have pointed out that the myocardial infarction leads to the necrosis and dissolution of abundant myocardial cells as well as the infiltration of inflammatory cells, which further causes the decreased functioning of the heart (24). The myocardial infarction results in the abnormal intracellular environment due to the insufficient energy supply (25). The increased compensatory contraction of the heart could cause the elevation in the level of the reactive oxygen species (ROS) due to the NADPH. More severely, the elevation of the ROS level will trigger the mitochondria to produce abundant ROS (the mechanism of 'oxygen triggering oxygen releasing' inside the mitochondria) (26). Oxidative stress will not only attack the cell membrane and organelle, but even interact with inflammatory factors to initiate inflammatory responses, further aggravating the myocardial damage caused by the infarction (27). So increasing the oxidase level and lowering the content of ROS are often seen as an important instruction to treat the myocardial infarction (28). In this study, we found that isoquercetin significantly reduced oxidative stress, iNOS expression and heart cell apoptosis, and induced eNOS expression in a rat with AMI. Lu et al showed that isoquercetin ameliorates tunicamycin-induced apoptosis through suppressing ROS-dependent stress (18).

It has been reported that the TLR4 receptor in the myocytes could be activated by the ligands PAMP and DAMP (12). LPS from gram-negative bacteria is a typical TLR4 specificity PAMP ligand and often used as the instrumental medicine to activate TLR4 (29). HSP60 is the DAMP ligand of TLR4, derived from the myocardial ischemia. Studies have indicated that in the detached mouse cardiac myocytes, LPS could activate TLR4, increase the transcriptional activity of NF- $\mathrm{NB}$, induce the production of cellular factors and lower the contractility of myocytes (30). NF- $\kappa \mathrm{B}$ is a widely distributed nuclear transcription factor with extensive functions. It can be combined with the specific sequence in the promoter or enhancer regions of specific gene to initiate the transcription of the gene (30). Usually, $N F-\kappa B$ is combined with $\mathrm{I} \kappa \mathrm{B}$ to be an inactive alien complex existing in the cytoplasm (29). Under the effect of extracellular stimulation signals, I $\mathrm{B}$ is phosphorylated and then processed through ubiquitination (29). Based on the effect of $26 \mathrm{~S}$ protease, it will release $\mathrm{NF}-\kappa \mathrm{B}$ P50/P65 into the nucleus and combine with $\kappa \mathrm{B}$ on the target gene, leading to the conformation change in the DNA here and thus initiating or enhancing the target gene transcription (31). In the present study, we found that isoquercetin significantly suppressed TLR4 and $\mathrm{NF}-\kappa \mathrm{B}$ protein expression in MI rat. Wang et al showed that isoquercetin protects induced injury of cortical neurons in oxygen-glucose deprivation-reperfusion via suppression of TLR4-NF- $\kappa$ B signal pathway (32).

In conclusion, our data show isoquercetin has a clear role in modulating inflammation and oxidative stress and is effective for MI through TLR4-NF- $\mathrm{B}$ signal pathway. Further clinical research on the protective effects of isoquercetin on human MI cells is required.

\section{References}

1. Morita Y, Maeda K, Kondo T, Ishii H, Matsudaira K, Okumura N, Mitsuhashi H, Shibata R and Murohara T; Nagoya Acute Myocardial Infarction Study (NAMIS) Group: Impact of adiponectin and leptin on long-term adverse events in Japanese patients with acute myocardial infarction. Results from the Nagoya Acute Myocardial Infarction Study (NAMIS). Circ J 77: 2778-2785, 2013.

2. Jensen LJ, Munk K, Flyvbjerg A, Bøtker HE and Bjerre M: Soluble receptor of advanced glycation end-products in patients with acute myocardial infarction treated with remote ischaemic conditioning. Clin Lab 61: 323-328, 2015. 
3. Tanaka S, Masuda T, Kamiya K, Hamazaki N, Akiyama A Kamada Y, Maekawa E, Noda C, Yamaoka-Tojo M and Ako J: A Single session of neuromuscular electrical stimulation enhances vascular endothelial function and peripheral blood circulation in patients with acute myocardial infarction. Int Heart J 57: 676-681, 2016.

4. Høfsten DE, Kelbæk H, Helqvist S, Kløvgaard L, Holmvang L, Clemmensen P, Torp-Pedersen C, Tilsted HH, Bøtker HE, Jensen LO, et al: The third DANish study of optimal acute treatment of patients with ST-segment elevation myocardial infarction Ischemic postconditioning or deferred stent implantation versus conventional primary angioplasty and complete revascularization versus treatment of culprit lesion only: Rationale and design of the DANAMI 3 trial program. Am Heart J 169: 613-621, 2015

5. Uysal H and Ozcan S: The effect of individual education on patients' physical activity capacity after myocardial infarction. Int J Nurs Pract 21: 18-28, 2015.

6. Li Z, Li Y, Zhang T, Miao W and Su G: Comparison of the influence of ticagrelor and clopidogrel on inflammatory biomarkers and vascular endothelial function for patients with ST-segment elevation myocardial infarction receiving emergency percutaneous coronary intervention: Study protocol for a randomized controlled trial. Trials 17: 75, 2016.

7. Stefanadi E, Tousoulis D, Antoniades C, Katsi V, Bosinakou E, Vavuranakis E, Triantafyllou G, Marinou K, Tsioufis C, Papageorgiou N, et al: Early initiation of low-dose atorvastatin treatment after an acute ST-elevated myocardial infarction, decreases inflammatory process and prevents endothelial injury and activation. Int J Cardiol 133: 266-268, 2009.

8. Cheng L, Jin Z, Zhao R, Ren K, Deng C and Yu S: Resveratrol attenuates inflammation and oxidative stress induced by myocardial ischemia-reperfusion injury: Role of Nrf2/ARE pathway. Int J Clin Exp Med 8: 10420-10428, 2015.

9. Cheng XY, Gu XY, Gao Q, Zong QF, Li XH and Zhang Y: Effects of dexmedetomidine postconditioning on myocardial ischemia and the role of the PI3K/Akt-dependent signaling pathway in reperfusion injury. Mol Med Rep 14: 797-803, 2016.

10. Börekçi A, Gür M, Türkoğlu C, Selek Ş, Baykan AO, Şeker T, Harbalıoglu H, Özaltun B, Makça İ, Aksoy N, et al: Oxidative stress and spontaneous reperfusion of infarct-related artery in patients with ST-segment elevation myocardial infarction. Clin Appl Thromb Hemost 22: 171-177, 2016.

11. Elmas E, Ahmad-Nejad P, Weiss C, Neumaier $M$ and Borggrefe M: Plasminogen activator inhibitor-1 (PAI-1), toll-like receptor 4 (TLR4), factor II (FII), FXIII and fibrinogen polymorphisms are not associated with the prevalence of sudden death due to ventricular fibrillation during myocardial infarction. Clin Chem Lab Med 46: 1329-1331, 2008.

12. Liu Q, Zhang J, Xu Y, Huang Y and Wu C: Effect of carvedilol on cardiomyocyte apoptosis in a rat model of myocardial infarction: A role for toll-like receptor 4. Indian $\mathrm{J}$ Pharmacol 45: 458-463, 2013.

13. Yang Y, Lv J, Jiang S, Ma Z, Wang D, Hu W, Deng C, Fan C, Di S, Sun Y and Yi W: The emerging role of Toll-like receptor 4 in myocardial inflammation. Cell Death Dis 7: e2234, 2016.

14. Wang CP, Shi YW, Tang M, Zhang XC, Gu Y, Liang XM, Wang ZW and Ding F: Isoquercetin ameliorates cerebral impairment in focal ischemia through anti-oxidative, anti-inflammatory and anti-apoptotic effects in primary culture of rat hippocampal neurons and hippocampal CA1 region of rats. Mol Neurobiol 54: 2126-2142, 2017.

15. Bhatia N, Kaur G, Soni V, Kataria J and Dhawan RK: Evaluation of the wound healing potential of isoquercetin-based cream on scald burn injury in rats. Burns Trauma 4: 7, 2016.

16. Vongsak B, Sithisarn P and Gritsanapan W: Simultaneous determination of crypto-chlorogenic acid, isoquercetin, and astragalin contents in moringa oleifera leaf extracts by TLC-densitometric method. Evid Based Complement Alternat Med 2013: 917609, 2013.

17. Zhang R, Yao Y, Wang Y and Ren G: Antidiabetic activity of isoquercetin in diabetic KK-Ay mice. Nutr Metab (Lond) 8: 85, 2011.
18. Lu T, Zhang $\mathrm{C}$, Chai $\mathrm{M}$ and $\mathrm{An} \mathrm{Y}$ : Isoquercetin ameliorates tunicamycin-induced apoptosis in rat dorsal root ganglion neurons via suppressing ROS-dependent endoplasmic reticulum stress. Biomed Pharmacother 80: 343-351, 2016.

19. Mao S, Wang L, Ouyang W, Zhou Y, Qi J, Guo L, Zhang M and Hinek A: Traditional Chinese medicine, Danlou tablets alleviate adverse left ventricular remodeling after myocardial infarction: Results of a double-blind, randomized, placebo-controlled, pilot study. BMC Complement Altern Med 16: 447, 2016.

20. Eitel I, Pöss J, Jobs A, Eitel C, de Waha S, Barkhausen J, Desch S and Thiele H: Left ventricular global function index assessed by cardiovascular magnetic resonance for the prediction of cardiovascular events in ST-elevation myocardial infarction. J Cardiovasc Magn Reson 17: 62, 2015.

21. Ketchum ES, Dickstein K, Kjekshus J, Pitt B, Wong MF, Linker DT and Levy WC: The Seattle Post Myocardial Infarction Model (SPIM): Prediction of mortality after acute myocardial infarction with left ventricular dysfunction. Eur Heart J Acute Cardiovasc Care 3: 46-55, 2014.

22. Kohl LP, Leimberger JD, Chiswell K, Jones WS, Thiele H, Smalling RW, Chandra P, Cohen M, Perera D, Chew DP, et al: Clinical characteristics and outcomes after unplanned intraaortic balloon counterpulsation in the counterpulsation to reduce infarct size Pre-PCI acute myocardial infarction trial. Am Heart J 174: 7-13, 2016

23. Prondzinsky R, Unverzagt S, Lemm H, Wegener N, Heinroth K, Buerke U, Fiedler M, Thiery J, Haerting J, Werdan K and Buerke M: Acute myocardial infarction and cardiogenic shock: Prognostic impact of cytokines: INF- $\gamma$, TNF- $\alpha$, MIP-1 $\beta$, G-CSF, and MCP-1 $\beta$. Med Klin Intensivmed Notfmed 107: 476-484, 2012.

24. Raish M: Momordica charantia polysaccharides ameliorate oxidative stress, hyperlipidemia, inflammation, and apoptosis during myocardial infarction by inhibiting the NF- $\mathrm{BB}$ signaling pathway. Int J Biol Macromol 97: 544-551, 2017.

25. Basili S, Tanzilli G, Mangieri E, Raparelli V, Di Santo S, Pignatelli $\mathrm{P}$ and Violi F: Intravenous ascorbic acid infusion improves myocardial perfusion grade during elective percutaneous coronary intervention: Relationship with oxidative stress markers. JACC Cardiovasc Interv 3: 221-229, 2010.

26. Aldakkak M, Stowe DF, Heisner JS, Riess ML and Camara AK: Adding ROS quenchers to cold $\mathrm{K}+$ cardioplegia reduces superoxide emission during 2-hour global cold cardiac ischemia. J Cardiovasc Pharmacol Ther 17: 93-101, 2012.

27. Feng Q, Lu C, Wang L, Song L, Li C and Uppada RC: Effects of renal denervation on cardiac oxidative stress and local activity of the sympathetic nervous system and renin-angiotensin system in acute myocardial infracted dogs. BMC Cardiovasc Disord 17: 65, 2017.

28. Sedlic F, Muravyeva MY, Sepac A, Sedlic M, Williams AM, Yang M, Bai X and Bosnjak ZJ: Targeted modification of mitochondrial ROS production converts high glucose-induced cytotoxicity to cytoprotection: Effects on anesthetic preconditioning. J Cell Physiol 232: 216-224, 2017.

29. Lin J, Wang H, Li J, Wang Q, Zhang S, Feng N, Fan R and Pei J: $\kappa-O p i o i d ~ r e c e p t o r$ stimulation modulates TLR4/NF- $\kappa \mathrm{B}$ signaling in the rat heart subjected to ischemia-reperfusion. Cytokine 61: 842-848, 2013.

30. Sun Y, Huang $J$ and Song K: BET protein inhibition mitigates acute myocardial infarction damage in rats via the TLR4/TRAF6/NF- $\kappa$ B pathway. Exp Ther Med 10: 2319-2324, 2015.

31. Wang Y, Li C, Cheng K, Zhang R, Narsinh K, Li S, Li X, Qin X, Zhang R, Li C, et al: Activation of liver X receptor improves viability of adipose-derived mesenchymal stem cells to attenuate myocardial ischemia injury through TLR4/NF- $\kappa \mathrm{B}$ and Keap-1/Nrf-2 signaling pathways. Antioxid Redox Signal 21: 2543-2557, 2014.

32. Wang CP, Li JL, Zhang LZ, Zhang XC, Yu S, Liang XM, Ding F and Wang ZW: Isoquercetin protects cortical neurons from oxygen-glucose deprivation-reperfusion induced injury via suppression of TLR4-NF- $\kappa$ B, signal pathway. Neurochem Int 63: 741-749, 2013. 